\title{
The Accuracy of 4D Ultrasonography in Determining Fetal Behavior
}

\author{
Nazeeha Waseem* \\ Department of Radiological sciences and Medical imaging, University of Lahore Pakistan. \\ PO box 50700 Gujrat, Punjab, Pakistan \\ E-mail: drnazeehawaseem@gmail.com \\ Bakhtawer \\ Zoology Department, University of Gujrat \\ Abid Ali \\ Department of Allied Health Sciences, University of Lahore Pakistan \\ Umair Waqas \\ Department of Allied Health Sciences, University of Lahore Pakistan
}

\begin{abstract}
Ultrasonography has been used for decades as a reliable diagnostic tool to detect fetal anomalies. Aside from technological improvements, image quality has become significantly better. The latest form of fetal ultrasonography is four-dimensional (4D) ultrasound that provides real-time imaging capabilities with impressive image quality. As 4D ultrasonography is becoming widely used in the perinatal screening of fetal anomalies and detection of fetal behavior, the debate of this device's accuracy is still ongoing. One key factor that determines the accuracy of 4D ultrasonography in assessing fetal behavior is the experience of the user in fields of fetal anomalies and the associated behavioral characteristics. This paper will tackle the topic of 4D ultrasound accuracy in detecting fetal behavior and screening for underlying pathologies.
\end{abstract}

Keywords: fetal behavior, fetal anomaly, 4D ultrasonography

DOI: $10.7176 / \mathrm{JHMN} / 80-02$

Publication date: September $30^{\text {th }} 2020$

\section{Introduction}

Fetal movement and behavior are important ultrasonographic characteristics that could inform the clinicians about several structural and physiological functions of the fetus. (1)

As scientists became more aware of fetal growth and embryological phases, the need for objective evaluation of the fetus during the perinatal phase has become crucial to address some preventable complications. (2) However, this task was challenging due to the exceptional susceptibility of fetuses to most X-ray beams used in the field of radiology. (3)

When the ultrasound machine was first introduced, healthcare professionals were skeptical about the effects of the sound waves on fetal development and organogenesis; however, and after several decades of research and clinical application, ultrasound machines seem to be completely safe. (4)

At first, these devices were mere black and white images that look like random chalk drawings to the nontrained eye. The quality of the images and accuracy of these devices were also mediocre, especially when dealing with low frequencies and deeper tissues.

As the technology of ultrasound advanced, the new machines provide high-quality images with exceptional accuracy, which allowed physicians to detect fetal abnormalities early on in the course of the pregnancy. (5) These devices facilitated the objective evaluation of fetal behavior and movement to screen for any abnormal finding and address the issue accordingly.

In this paper, we will comprehensively cover the technology of 4D ultrasonography, its role in detecting fetal behavior, and the accuracy of the collected findings.

\section{4d Ultrasonography}

Unlike 3D ultrasound, which allows the observation of fetal anatomical structures as static 3D images (6), 4D ultrasonography provides clinicians with live streaming videos of the images that show the motion of fetal heart (e.g., endocardium, valves, blood flow). In simpler terms, this machine adds motion to the static images produced by $3 \mathrm{D}$ ultrasound. It uses either a $2 \mathrm{D}$ transducer that rapidly acquires 20-30 volumes or a matrix array $3 \mathrm{D}$ transducer is used. (7)

When it comes to advantages, 4D ultrasound offers similar features as the older model (i.e., 3D ultrasonography) with the added motion to various hollow organs. As of the time being, researchers are still 
experimenting to decipher the clinical value of this technology relative to older models. (8)

The primary disadvantages to $4 \mathrm{D}$ ultrasound include the production of higher levels of energy with potential side effects to the fetus, the longer duration of ultrasound sessions, and the unreliable results when the operator did not receive professional training in this field (using the 4D ultrasound machine does not require a certificate). (9)

\section{Fetal behavior during early weeks}

Fetal movements are generally felt by pregnant women as a sign of development in size and strength. Initially, these movements are uniquely felt by the pregnant woman; however, and over time, others can also perceive them. (10)

Oftentimes, healthcare professionals advise being aware of these movements, as they transmit a sign of fetal well-being.

For instance, a reduced fetal movement can be a warning sign of fetal impairment, which warrants further investigation by the healthcare provider.

The very first fetal movements felt by the pregnant woman are generally called quickening, which can be considered a warning sign to the woman that she has a fetus growing inside of her uterus. (11)

The timing of quickening is typically limited between the $16^{\text {th }}$ and the $22^{\text {nd }}$ week of gestation. In addition to these movements, the pregnant woman will experience other presumptive signs of pregnancy, such as flatus, peristalsis, and abdominal cramping. (12)

Note that multiparous mothers generally sense fetal movements earlier than primiparous women by an interval of 4-6 weeks. In most cases, the healthcare professional will be able to externally sense these movements around the $20^{\text {th }}$ week of gestation, which is considered a positive sign of pregnancy. (13)

During the third trimester, however, being aware of these movements becomes even more critical, as it's the time windows where several structural and physiological functions go array. Most doctors will recommend keeping a notebook to document the movements of the fetus, their nature, and any fluctuating patterns. (14)

The primary sign to look for is a decrease in fetal movements, which historically, have been linked to fetal demise. Therefore, the assumption is that if a woman notices a decrease in fetal movement, there is an adverse event with the fetus that can be avoided by the proper interventions. (15)

Several studies aimed to confirm the connection between decreased fetal movements and some disrupted physiological parameters, such as placental functioning, uterus abnormalities, fetal growth restriction, tight nuchal cords, and fetal demise, in an attempt to prevent these complications before they occur. (16) (17) (18)

According to one study, researchers demonstrated a connection between the high incidence of poor fetal prognosis and decreased fetal movement during the third trimester. (19)

Consequently, healthcare organizations released a series of guidelines that indicate an urgent evaluation of pregnant women who notice decreased fetal movement.

However, these guidelines do not come without problems, as the increased number of prenatal visits could place a toll on the healthcare system. (20)

The healthcare provider needs to remember that some pregnant women are better (i.e., more vigilant) at detecting fetal movements than others. For this reason, there is a dire need for other objective methods that can convey relevant clinical results of the fetus' anatomy and physiology.

This is where the role of ultrasound becomes apparent, as it provides relevant and reliable data about the intrauterine cavity, fetal movements, organ development, and behavioral characteristics.

\section{The assessment of fetal behavior of growth-restricted fetuses by 4D sonography}

The primary source that allowed researchers to understand the development of fetal motor behavior has been 4D ultrasound, which can offer real-time visualization of the fetus or videotapes for later observation. (21)

One study attempted to assess the possibility of observing the dynamic movements of the fetus during the first and second trimesters using different imaging techniques and concluded that It's not feasible without the technology of 4D ultrasound. Additionally, clinicians can also obtain significantly higher image quality, as the 4D ultrasound allows the visualization of the whole fetus. (22)

The identification of measurable indicators of fetal behavior that mirror the development of the central nervous system, as well as the peripheral nervous system, is one of the primary goals of fetal neurobehavioral research. (23) However, there are no unified assessment methods to evaluate fetal neurobehavioral development.

The importance of ultrasound is based on the currently believed theories that neurobehavioral functioning measured in full-term neonates and infants does not start after birth but rather during the intrauterine phase, which further emphasizes the importance of 4D ultrasound.

With that being said, the construction of a unified fetal neurobehavioral assessment during the intrauterine phase is not warranted at the moment due to scarce normative data that document the prognosis of these babies.

For instance, factors that precipitate intrauterine growth retardation (IUGR) also boost the process of 
neuromaturation, which excludes the "all-or-nothing" aspect from the equation. Evidence of accelerated neuromaturation "as a progressive response by variable degree" in IUGR pregnancies is also seen with auditory evoked responses. (24)

According to Pettigrew and coworkers, when analyzing the mean conduction times in 25 preterm small for gestational age (SGA) infants and 76 appropriate for gestational age (AGA) infants, the first group had significantly shorter conduction time in brainstem auditory evoked responses (i.e., a higher degree of neuromaturation). (25)

Furthermore, Henderson-Smart confirmed this finding by stating that preterm AGA infants born to hypertensive mothers had mean conduction times between those of SGA and AGA infants born to nonhypertensive mothers. (26) However, peripheral nerve conduction times are similar in AGA and SGA infants.

In one study, scientists analyzed 6 facial expressions, 5 hand movements, and 3 head movements, and noticed that fetuses with IUGR are less active compared to control groups. (27)

In another study, Roodenburg noted that inhibition is an extremely important sign of central nervous system development and that most research reports less activity as the fetus becomes older. According to resources, advanced gestational age makes fetuses move one time every minute. (28)

When we reach the third trimester, the "hand-to-head" movement becomes predominantly more prevalent than other movement patterns. While the fetus is able to move his hands to other body parts, the only time we consider this movement to be intentional is during the postnatal period.

By the $32^{\text {nd }}$ week, the "hand away from the body" movements are predominant, which reflects tactile proclivity and neural maturation.

An additional difference that occurs after the $37^{\text {th }}$ week is the reduced frequency of the "hand to face" or "hand to head" movement. Researchers hypothesize that this behavior aims to conserve energy in preparation for birth.

Significant trends in fetal eye movement organization can also be observed during the second half of pregnancy, especially during the third trimester, and eye movement patterns sensitively reflect the activity of the neural control system. The earliest eye movements appear at 16-18 weeks of gestation, as sporadic movements with a limited frequency. Moreover, some movement patterns occurred more frequently than others. (29)

Researchers stated that isolated eye blinking patterns appear more frequently and begin to consolidate at 24-26 weeks' gestation. (30)

One study found a slight difference in the frequency of isolated eye blinking between normal and IUGR fetuses. Those IUGR fetuses seem to blink the eyes less frequently than normal fetuses. (31)

Scientists also noted characteristic behavioral patterns in fetuses with IUGR. In fact, 11 cases of IUGF showed a poor repertoire of fetal behavioral patterns (e.g., 3 have abnormal cardiotocography findings, 4 affected by preeclampsia, 1 has periventricular hematoma, 1 has meconium). (32)

According to Ferrari and Prechtl, monotony and lack of complex movement sequences are the hallmarks of a poor repertoire. (33)

\section{The accuracy of 4D ultrasonography in determining fetal behavior}

Researchers are still debating about the accuracy of 4D ultrasonography in detecting fetal behavior to predict the prognosis of these fetuses and plan a therapeutic approach. (34)

However, and due to the relative freshness of this technology, there is scarce evidence to confirm or revoke its accuracy.

As discussed above, numerous behavioral patterns can be objectively identified by the 4D ultrasound machine, which can later be dissected after recording the tape to analyze every frame of the video and aim to detect abnormal behavior. For instance, eye and head movements are excellent predictors of brain development and peripheral motion. (35)

Additionally, experts believe that monitoring fetal behavior during the perinatal phase can predict the development of the central nervous system and the peripheral nervous system, as well as to detect any structural or functional abnormalities. This allows neuro-pediatrician to detect early perinatal neurological damage and develop appropriate treatment plans. (36) 

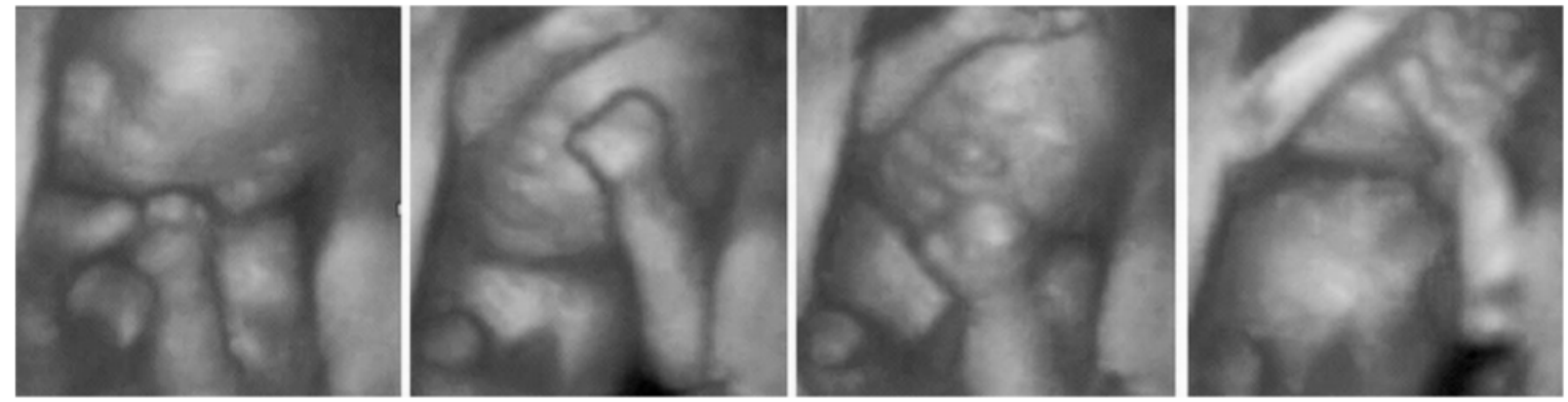

Figure $14 D$ sequence that depicts fetal movements in various directions. The most notable patterns is the head movement followed by opening both palms. (37)
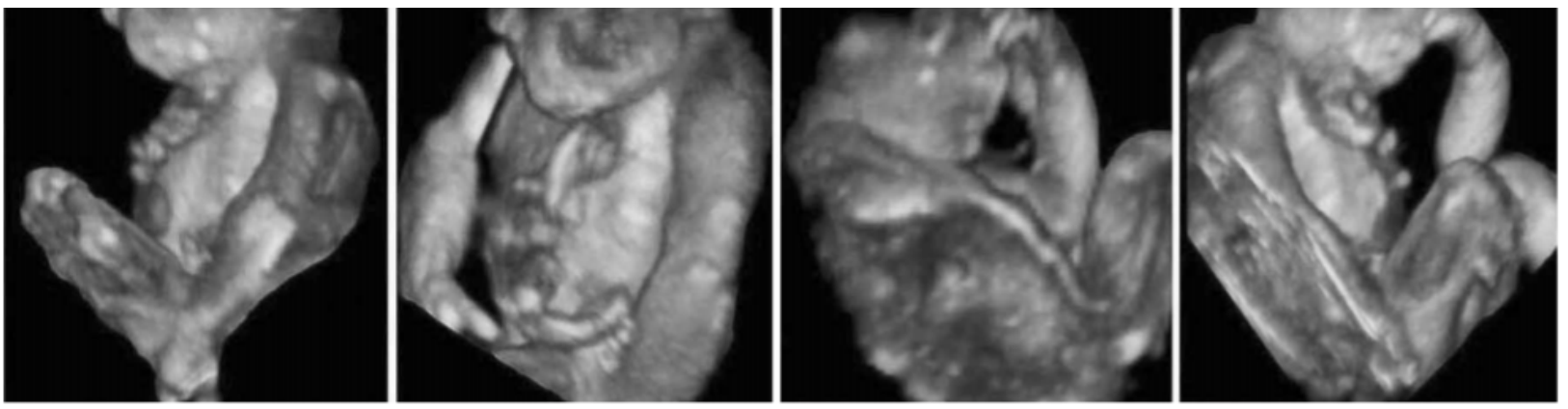

Figure $24 D$ sequence depicting an anencephalic fetus that engages in unidirectional movement (e.g., hand to head). This sequence also confirms the lack of changes in positions. (37)

Figures 1 and 2 demonstrate the behavioral pattern of the anencephalic fetus and showcases the characteristic head and hand movements.

The accuracy and reliability of these devices in determining fetal behavior has been a topic of debate for several years with two schools of thoughts; the first group believes that 4D ultrasound cannot be uniquely relied on to come up with any conclusions about the neurobehavioral developments of the future, as well as its prognosis. On the other hand, the second group advocates for the use of 4D ultrasonography in the field of perinatal screening for disease in order to organize a precocious plan that improves the outcome of these babies. (38)

In summary, there is not sufficient evidence to support any of these hypotheses, but researchers are optimistic about the potential uses of 4D ultrasonography in the field of obstetrics. (39)

We conclude that further research is warranted to objectively assess the accuracy of 4D ultrasound in determining fetal behavior.

\section{Conclusion}

The 4D ultrasound technology is a fantastic tool that allowed physicians to detect fetal development, which improved the overall prognosis of fetuses with serious anatomical defects and also helped in the prediction of neurobehavioral abnormalities.

The use of 4D ultrasound to get real-time images of the fetus' movement and behavior in response to different internal and external stimuli is expected to reach more areas around the world to increase the efficiency of prenatal and perinatal screening in the detection of any abnormal findings.

When it comes to accuracy and reliability of 4D ultrasound in detecting fetal behavioral abnormalities, the data is scarce, and search is highly needed.

\section{References}

1- Bryant, J., Jamil, R. T., \& Thistle, J. (2019). Fetal Movement. In StatPearls [Internet]. StatPearls Publishing.

2- Rayburn, W. F., Jolley, J. A., \& Simpson, L. L. (2015). Advances in ultrasound imaging for congenital malformations during early gestation. Birth Defects Research Part A: Clinical and Molecular Teratology, 103(4), 260-268.

3- Chaparian, A., \& Aghabagheri, M. (2013). Fetal radiation doses and subsequent risks from X-ray examinations: Should we be concerned?. Iranian Journal of Reproductive Medicine, 11(11), 899.

4- Torloni, M. R., Vedmedovska, N., Merialdi, M., Betrán, A. P., Allen, T., González, R., Platt, L. D., \& ISUOG-WHO Fetal Growth Study Group (2009). Safety of ultrasonography in pregnancy: WHO systematic 
review of the literature and meta-analysis. Ultrasound in obstetrics \& gynecology : the official journal of the International Society of Ultrasound in Obstetrics and Gynecology, 33(5), 599-608. https://doi.org/10.1002/uog.6328

5- Abramowicz, J. S. (2013, October). Benefits and risks of ultrasound in pregnancy. In Seminars in perinatology (Vol. 37, No. 5, pp. 295-300). WB Saunders.

6- Dimitrova, V., Markov, D., \& Dimitrov, R. (2007). 3D and 4D ultrasonography in obstetrics. Akusherstvo i ginekologiia, 46(2), 31-40.

7- Achiron, R., Gindes, L., Zalel, Y., Lipitz, S., \& Weisz, B. (2008). Three-and four-dimensional ultrasound: new methods for evaluating fetal thoracic anomalies. Ultrasound in Obstetrics and Gynecology: The Official Journal of the International Society of Ultrasound in Obstetrics and Gynecology, 32(1), 36-43.

8- Pooh, R. K., Maeda, K., Kurjak, A., Sen, C., Ebrashy, A., Adra, A., ... \& Stanojevic, M. (2016). 3D/4D sonography-any safety problem. Journal of Perinatal Medicine, 44(2), 125-129.

9- Haar, G. T. (2011). Ultrasonic imaging: safety considerations. Interface focus, 1(4), 686-697.

10- Bryant, J., Jamil, R. T., \& Thistle, J. (2019). Fetal Movement. In StatPearls [Internet]. StatPearls Publishing.

11- KRAUS, G. W., \& HENDRICKS, C. H. (1964). Significance of the quickening date in determining duration of pregnancy. Obstetrics \& Gynecology, 24(2), 178-182.

12- Foxcroft, K. F., Callaway, L. K., Byrne, N. M., \& Webster, J. (2013). Development and validation of a pregnancy symptoms inventory. BMC pregnancy and childbirth, 13(1), 3.

13- Bryant, J., Jamil, R. T., \& Thistle, J. (2019). Fetal Movement. In StatPearls [Internet]. StatPearls Publishing.

14- Sheikh, M., Hantoushzadeh, S., \& Shariat, M. (2014). Maternal perception of decreased fetal movements from maternal and fetal perspectives, a cohort study. BMC pregnancy and childbirth, 14(1), 286.

15-Delaram, M., \& Jafarzadeh, L. (2016). The effects of fetal movement counting on pregnancy outcomes. Journal of clinical and diagnostic research: JCDR, 10(2), SC22.

16- Tuffnell, D. J., Cartmill, R. S., \& Lilford, R. J. (1991). Fetal movements; factors affecting their perception. European Journal of Obstetrics \& Gynecology and Reproductive Biology, 39(3), 165-167.

17- Daly, L. M., Gardener, G., Bowring, V., Burton, W., Chadha, Y., Ellwood, D., ... \& McDonald, S. (2018). Care of pregnant women with decreased fetal movements: Update of a clinical practice guideline for Australia and New Zealand. Australian and New Zealand Journal of Obstetrics and Gynaecology, 58(4), 463-468.

18- Frøen, J. F., Tveit, J. V. H., Saastad, E., Børdahl, P. E., Stray-Pedersen, B., Heazell, A. E., ... \& Fretts, R. C. (2008, August). Management of decreased fetal movements. In Seminars in perinatology (Vol. 32, No. 4, pp. 307-311). WB Saunders.

19- Huang, C., Han, W., \& Fan, Y. (2019). Correlation study between increased fetal movement during the third trimester and neonatal outcome. BMC Pregnancy and Childbirth, 19(1), 467.

20- Carter, E. B., Tuuli, M. G., Caughey, A. B., Odibo, A. O., Macones, G. A., \& Cahill, A. G. (2016). Number of prenatal visits and pregnancy outcomes in low-risk women. Journal of perinatology, 36(3), 178-181.

21- Lebit, F. D., \& Vladareanu, R. (2011). The role of 4D ultrasound in the assessment of fetal behaviour. Mædica, 6(2), 120.

22- Kurjak, A., Miskovic, B., Andonotopo, W., Stanojevic, M., Azumendi, G., \& Vrcic, H. (2007). How useful is 3D and 4D ultrasound in perinatal medicine?. Journal of perinatal medicine, 35(1), 10-27.

23- Hata, T. (2016). Current status of fetal neurodevelopmental assessment: Four-dimensional ultrasound study. Journal of Obstetrics and Gynaecology Research, 42(10), 1211-1221.

24- Rodgers, L., \& Furcron, C. (2016). The dynamic interface between neuromaturation, risky behavior, creative dance movement, and youth development programming. American Journal of Dance Therapy, 38(1), 3-20.

25- Kittler, P. M., Phan, H. T., Gardner, J. M., Miroshnichenko, I., Gordon, A., \& Karmel, B. Z. (2009). Auditory brainstem evoked responses in newborns with Down syndrome. American journal on intellectual and developmental disabilities, 114(6), 393-400.

26- De Jesus, L. C., Pappas, A., Shankaran, S., Li, L., Das, A., Bell, E. F., ... \& Newman, N. S. (2013). Outcomes of small for gestational age infants born at $<27$ weeks' gestation. The Journal of pediatrics, 163(1), 55-60.

27- Kurjak, A., Predojevic, M., Stanojevic, M., Salihagic-Kadic, A., Miskovic, B., Badreldeen, A., ... \& Honemeyer, U. (2010). Intrauterine growth restriction and cerebral palsy. Acta Informatica Medica, 18(2), 64.

28- Morokuma, S., Fukushima, K., Kawai, N., Tomonaga, M., Satoh, S., \& Nakano, H. (2004). Fetal habituation correlates with functional brain development. Behavioural Brain Research, 153(2), 459-463.

29- Okawa, H., Morokuma, S., Maehara, K., Arata, A., Ohmura, Y., Horinouchi, T., ... \& Kato, K. (2017). Eye movement activity in normal human fetuses between 24 and 39 weeks of gestation. Plos one, 12(7), e0178722.

30- Horimoto, N., Hepper, P. G., Shahidullah, S., \& Koyanagi, T. (1993). Fetal eye movements. Ultrasound in 
Obstetrics and Gynecology: The Official Journal of the International Society of Ultrasound in Obstetrics and Gynecology, 3(5), 362-369.

31- Kurjak, A., Predojevic, M., Stanojevic, M., Talic, A., Honemeyer, U., \& Kadic, A. S. (2011). The use of 4D imaging in the behavioral assessment of high-risk fetuses. Imaging in Medicine, 3(5), 557.

32- Beccaria, E., Martino, M., Briatore, E., Podestà, B., Pomero, G., Micciolo, R., ... \& Calzolari, S. (2012). Poor repertoire General Movements predict some aspects of development outcome at 2 years in very preterm infants. Early human development, 88(6), 393-396.

33- Nakajima, Y., Einspieler, C., Marschik, P. B., Bos, A. F., \& Prechtl, H. F. (2006). Does a detailed assessment of poor repertoire general movements help to identify those infants who will develop normally?. Early human development, 82(1), 53-59.

34-Kurjak, A., Stanojević, M., Salihagić-Kadić, A., Spalldi Barišić, L., \& Jakovljević, M. (2019). Is FourDimensional (4D) Ultrasound Entering a New Field of Fetal Psychiatry?. Psychiatria Danubina, 31(2), 133140 .

35-AboEllail, M. A. M., Kanenishi, K., Mori, N., Mohamed, O. A. K., \& Hata, T. (2018). 4D ultrasound study of fetal facial expressions in the third trimester of pregnancy. The Journal of Maternal-Fetal \& Neonatal Medicine, 31(14), 1856-1864.

36- Lai, J., Nowlan, N. C., Vaidyanathan, R., Shaw, C. J., \& Lees, C. C. (2016). Fetal movements as a predictor of health. Acta obstetricia et gynecologica Scandinavica, 95(9), 968-975.

37- Andonotopo, W., Kurjak, A., \& Kosuta, M. I. (2005). Behavior of an anencephalic fetus studied by 4D sonography. The Journal of Maternal-Fetal \& Neonatal Medicine, 17(2), 165-168.

38- DiPietro, J. A., Costigan, K. A., \& Voegtline, K. M. (2015). Studies in fetal behavior: revisited, renewed, and reimagined. Monographs of the Society for Research in Child Development, 80(3), vii.

39- Hata, T., Kurjak, A., \& Kozuma, S. (Eds.). (2012). Current topics on fetal 3D/4D ultrasound. Bentham Science Publishers. 